\title{
Navigation by Dead Reckoning and Local Cues
}

\author{
A. S. Etienne and V. Séguinot
}

(University of Geneva)

I. INTRODUCTION. According to comprehensive theories of navigation, animals navigate by using two complementary strategies: (I) dead reckoning informs the subject in a continuous manner on its actual location with respect to an Earthbound or absolute coordinate system; while (2) long-term associations between particular landmarks and specific locations allow the animal to find its way within a familiar environment. If the subject structures familiar space as a system of interconnected places - the so-called 'cognitive map' - it may know through dead reckoning where it is located on its map and relate its route-based expectations to the actually perceived scenario of local cues. ${ }^{8,11}$

Our research is focused on homing strategies shown by golden hamsters in the hoarding situation. Throughout the test period, each of our experimental subjects lives alone in the test apparatus where it is provided with an adequate nest. It is only in these conditions that the hamsters may develop an intense and persistent motivation for hoarding on which we rely to test their homing behaviour. In a typical hoarding excursion, the hamster proceeds from its nest to a feeding place, where it fills its cheek pouches, and then returns home along the shortest path to deposit its load in its granary within its nest. If hoarding takes place under infrared (IR) light or in other conditions where no adequate optical references are available, homing occurs independently of any external cue, ${ }^{6}$ through 'internal' dead reckoning: during locomotion, the subject generates internal signals, which are continuously updated and therefore allow the animal to return home directly at any moment and from any point of its excursion. In contrast, if the subject is presented with familiar optical cues which it could previously associate with the location of the nest, it relies preferentially on these external references.

We deal with the following questions: ( $\mathrm{I} a$ ) what is the nature of the firstorder signals the animal derives from the angular and linear components of its locomotor activity, and ( $\mathrm{I} b$ ) how are these signals computed ${ }^{10}$ to yield the homing 'vector' ?; (2) on what conditions do hamsters rely on optical references rather than on internal dead reckoning, and what is the nature of good landmarks?; and finally, (3) can we maintain the cognitive map hypothesis on our subjects' level and assume that dead reckoning is an ubiquitous process, the outcome of which is automatically fed into the map system? According to an alternative view, our subjects may organize familiar space in a less coherent manner ${ }^{1}$ and use dead reckoning only to return to a particular point of departure 
rather than deriving from it their position within an allocentric frame of reference.

2. DEAD RECKONING: UPDATING THE ANGULAR AND LINEAR COMPONENTS OF LOCOMOTION. Figure I illustrates how our subjects home
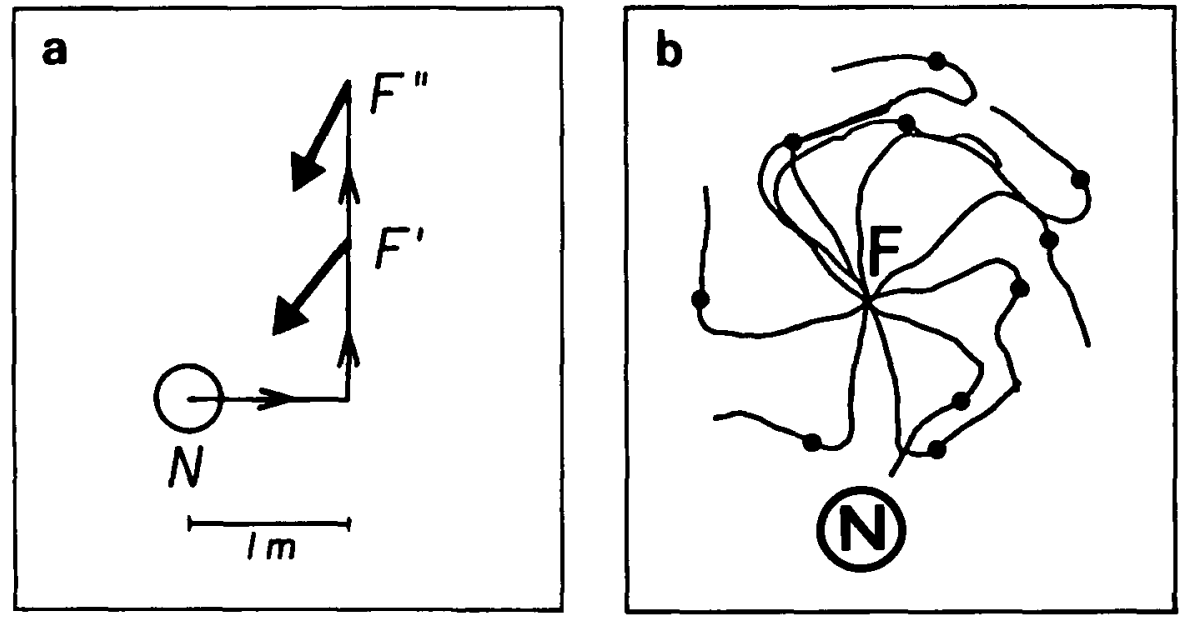

Fig. I. (a) Return direction after an L-shaped outward journey, under IR light. From its nest, $\mathrm{N}$, near the periphery of a square arena $(3.55 \times 3.55 \mathrm{~m})$, the subject is led along an $\mathrm{L}$-shaped detour to food source $\mathrm{F}^{\prime}$ or $\mathrm{F}^{\prime \prime}$, where it picks up food. The two vectors $(P<0.005$, Moore's test) that point to the nest represent the mean orientation of 5 subjects during their return to the nest, at a distance of $92 \mathrm{~cm}$ from $F^{\prime}$ or $F^{\prime \prime}$. Each subject was tested three times in each situation. (b) Homing behaviour of one subject in conditions of IR light and removal of the nest. The subject is led from its nest, N, along a direct path to a food source, F, on a horizontal platform, and the nest is removed from the test enclosure. During the uptake of food, the subject is slowly rotated. It is therefore induced to home in the wrong direction and to search for the nest without being guided by olfactory and other cues at the former nest location. The lines which irradiate from $F$ represent ten return journeys. The black dot indicates the first point of the return journey where the beeline distance from $F$ ceases to increase

by dead reckoning in conditions where they have to take into account the angular and linear components of the outward journey to the feeding place. After an Lshaped outward journey (graph (a)), all subjects returned by an adequate shortcut and therefore yielded (first- and second-order) vectors which point fairly directly towards the nest. This performance requires the moment-to-moment evaluation and processing of rotations and translations. In general, our results show that the homing direction is more sensitive to rotations than to translations. Furthermore, our hamsters, like other species, commit systematic homing errors, which may inform us on the algorithm these species use to update route-based signals. ${ }^{10}$

Graph (b) represents the typical homing behaviour of hamsters when their nest has been removed from the test enclosure. From the feeding place, the subject proceeds in a given direction along a straight path, until it has covered the approximate beeline distance between the feeding site and the former nest 
location. Failing to come across its (removed) nest, the animal then moves along the perimeter of a circle with a constant radius, thus maintaining a constant distance from the starting point of its return at the food source; along this circle, the subject moves opposite to the direction in which it has been rotated during the food uptake, as if it were correcting its initial homing direction. ${ }^{10}$ This and other results suggest that the subjects may not only compute the homing direction and distance (both of which require the estimation of rotations and translations) through separate processes, but also retrieve the corresponding information in separate steps during the performance of the return journey.

We are still far from knowing how species which home without using any external references measure both direction and distance. Experiments in which hamsters are rotated (passively) during the uptake of food show that vestibular signals originating from the stimulation of the semicircular canals play a role in the assessment of rotations, but that they need to be complemented by other categories of information. Indeed, hamsters cease to return from the centre of a circular arena towards their nest at the periphery after having been subjected to more than two or three full rotations; in contrast, they continue to choose a significant homing direction after having performed (actively) up to five closed loops around the arena centre before being given access to the food source. Nonvestibular information, such as somatosensory feedback, is likely to play an even greater role in the evaluation of the linear than in the assessment of the angular components of locomotion. Hamsters tend to undercompensate for passive translations, which act on the vestibular otoliths, and in certain conditions do not compensate for them at all. ${ }^{5,6}$ More specific, but mainly speculative hypotheses on the estimation of distance, involve a step-counting mechanism, the measure of the duration of locomotion, or the evaluation of correlated energy expenditure.

3. THE ROLE OF DEAD RECKONING AND VISUAL CUES IN CONFLICT SITUATIONS. To test the influence of optical references on the homing direction, the subjects live and are tested in a large circular arena with a peripheral nest. In standard conditions, the arena and visual cues from outside the arena remain in a constant position so that the animal can associate stable visual references with the nest entrance. In test conditions we either rotate the arena before the subject has left its nest, or present the optical cues in a changed angular position. Figure 2(a) illustrates two basic modalities of homing in experiments where the arena is rotated before the beginning of the outward journey. If the animals are tested under IR light, they return from the food source at the centre of the arena to the new, rotated position of the nest. In other words, they return to the point in absolute space where they started to update the outward journey through dead reckoning. If the same test takes place under light, so that the animals can see the distant optical environment, their homing trajectories are mainly directed towards the usual, standard nest location. Thus the animals rely predominantly on stable associations between visual configurations and the (invisible) nest entrance. At the same time, however, the return journeys are slightly deviated towards the new, rotated position of the nest and therefore express the influence of the dead-reckoning process, which always occurs. ${ }^{13}$ 

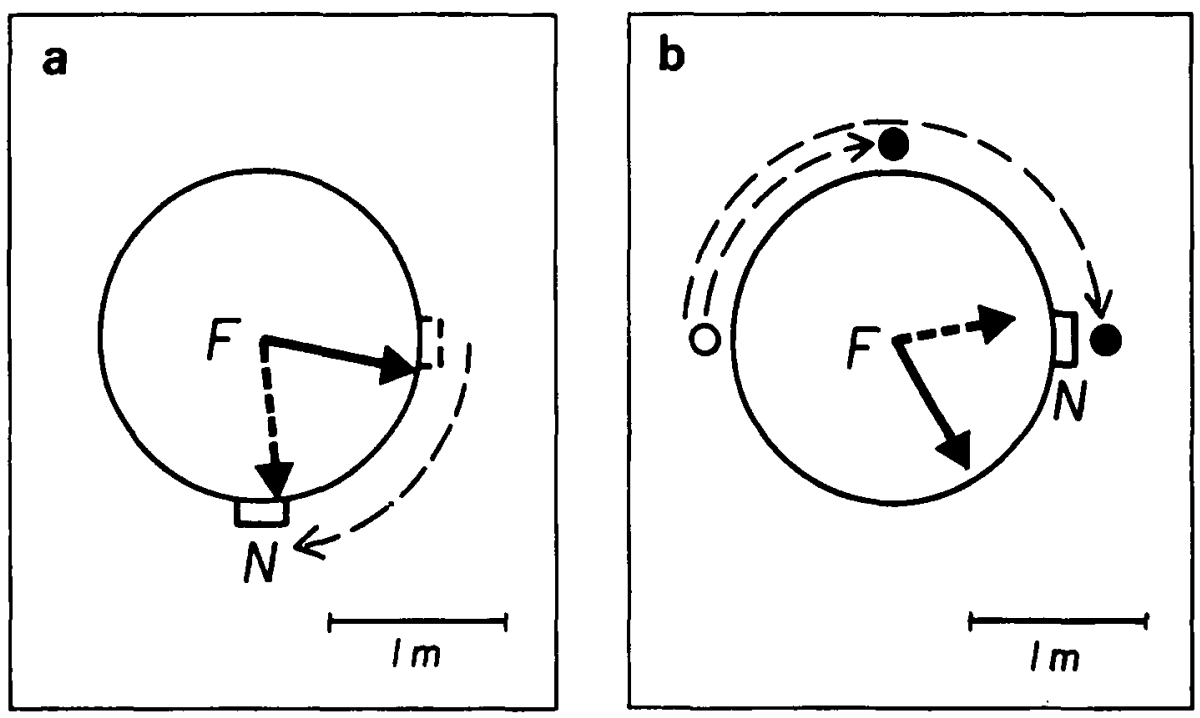

Fig. 2. (a) Return direction after rotation of arena and nest box in darkness and light. In its standard location the nest box (dashed rectangle) is located at $\circ^{\circ}$, on the right of a circular arena $\left(r=I^{\prime} \cdot 10 \mathrm{~m}\right)$. Here the arena and nest box, $N$, are rotated clockwise by $90^{\circ}$ before the subject leaves the nest to proceed to a food source, F, at the arena's centre. The dashed vector represents the mean orientation of the return itineraries to the periphery shown by 7 subjects tested under IR light; the solid vector represents the mean orientation of I I subjects tested under ordinary room light $(P<0.01$, Moore's test). Each subject underwent to trials. (b) Effect of a single visual cue on return direction. During the dark phase of the light-dark cycle, the hamster is exposed to a weak spot of light (small open circle) located opposite the nest, N. In the experimental trials, the animal leaves the nest from its standard position and proceeds under IR light to a food source, F, at the arena's centre. As it starts to pick up food, the spot is presented in an altered angular position (filled circles), where it remains until the subject has returned to the nest. The solid vector represents the mean orientation of the return trajectories of 12 animals tested under a $90^{\circ}$-clockwise shift of the spot $(P<0.0 \mathrm{I}$, Moore's test $)$. The dashed vector represents the mean orientation of 5 animals tested under a $180^{\circ}$ shift of the spot $(P<0.05$, Moore's test). Each subject underwent 10 trials

Experiments with a single visual cue - a dim light spot presented at a standard position just outside the arena - allowed us to analyse homing in a wide range of conflict situations between dead reckoning and a directional reference. In these experiments (see Fig. 2 (b)), the subjects proceed in total darkness from the nest exit to the feeding place. As soon as they reach the food source, the familiar light spot appears in a new angular position. In minor conflict situations, the homing direction follows mainly, but by no means totally, the rotation of the spot (see solid vector). In contrast, when the light spot and dead reckoning indicate two opposed directions, the subjects show greater variance in their behaviour, and a number of them rely predominantly on dead reckoning (see hatched vector). Thus hamsters tend to rely preferentially on distinctive visual cues, possibly because prominent features of the visual environment remain at a constant location, while dead reckoning is open to cumulative errors. However, when 
visual references seem less reliable they change over to dead reckoning, which is most likely an innate navigational strategy.

Whereas a weak light spot presented in darkness plays an important role as a stable directional reference, this is not necessarily the case for conspicuous visual configurations which are presented within a controlled visual environment. ${ }^{4}$ To examine the impact of a familiar visual scenario, the arena and nest are rotated before the beginning of the hoarding excursion. Thus the visual environment is set at variance with dead reckoning as described in Fig. 2 (a). So far, a number of experiments have given the following results. To predominate clearly over dead reckoning, the visual 'landscape' has to contain at least one vertical structure (such as a cylinder), which is superimposed on a continuous background pattern (see Fig. 3(a)). The background pattern presented alone, the vertical cylinder presented alone (graph (b)), or the same cylinder presented in a horizontal position against the continuous background pattern (graph (d)) exert a lesser influence on the homing direction than the above-mentioned combination (graph (c)). Our hypothesis that the depth dimension (vertical cylinder in the foreground and continuous pattern in the background) plays a decisive role was not confirmed. Neither could we show through the comparison between the effect of a vertically or horizontally striped card on the symmetrical enclosure that verticality in general has a greater impact than horizontally. Thus, it appears to be an extremely difficult matter to solve a long-standing problem in the psychological and neurophysiological study of spatial behaviour; namely, to determine the optical parameters of 'good' landmarks from outside the test apparatus.

Experiments are in progress which show that hamsters easily learn to use three-dimensional structures on the arena floor to find a food source ${ }^{2}$ and to return to the nest, and that in our test conditions these structures within the arena play a greater role than visual configurations from outside the arena.

4. THE UBIQUITY OF DEAD RECKONING AND ITS INTERRELATION WITH THE COGNITIVE MAP. The question as to whether dead reckoning plays a general and necessary role in navigation, and how it interacts with the subject's representation of familiar space, is approached through a variety of different situations. As far as the generality of dead reckoning is concerned, we tested the hamster's capacity to return not only from the feeding place to its nest, but also from its nest to a newly discovered food source without relying on external references. Our second-order data show that the subjects return significantly to the last finding site in conditions where only a twofold dead-reckoning process can explain the animals' commutation between the nest and a feeding place : the 'integration' of the first outward journey from the nest to a new finding site yields the necessary information for the direct return to the nest, and the integration of the first return to the nest yields the information for the second visit to the (last) finding site. However, according to all our first-order data, the return to the nest is always much more precise than the (first) return to a particular feeding place - a fact which casts some doubt on the tempting assumption that central place foragers may go back and forth between a new profitable food source and their nest through dead reckoning only. 


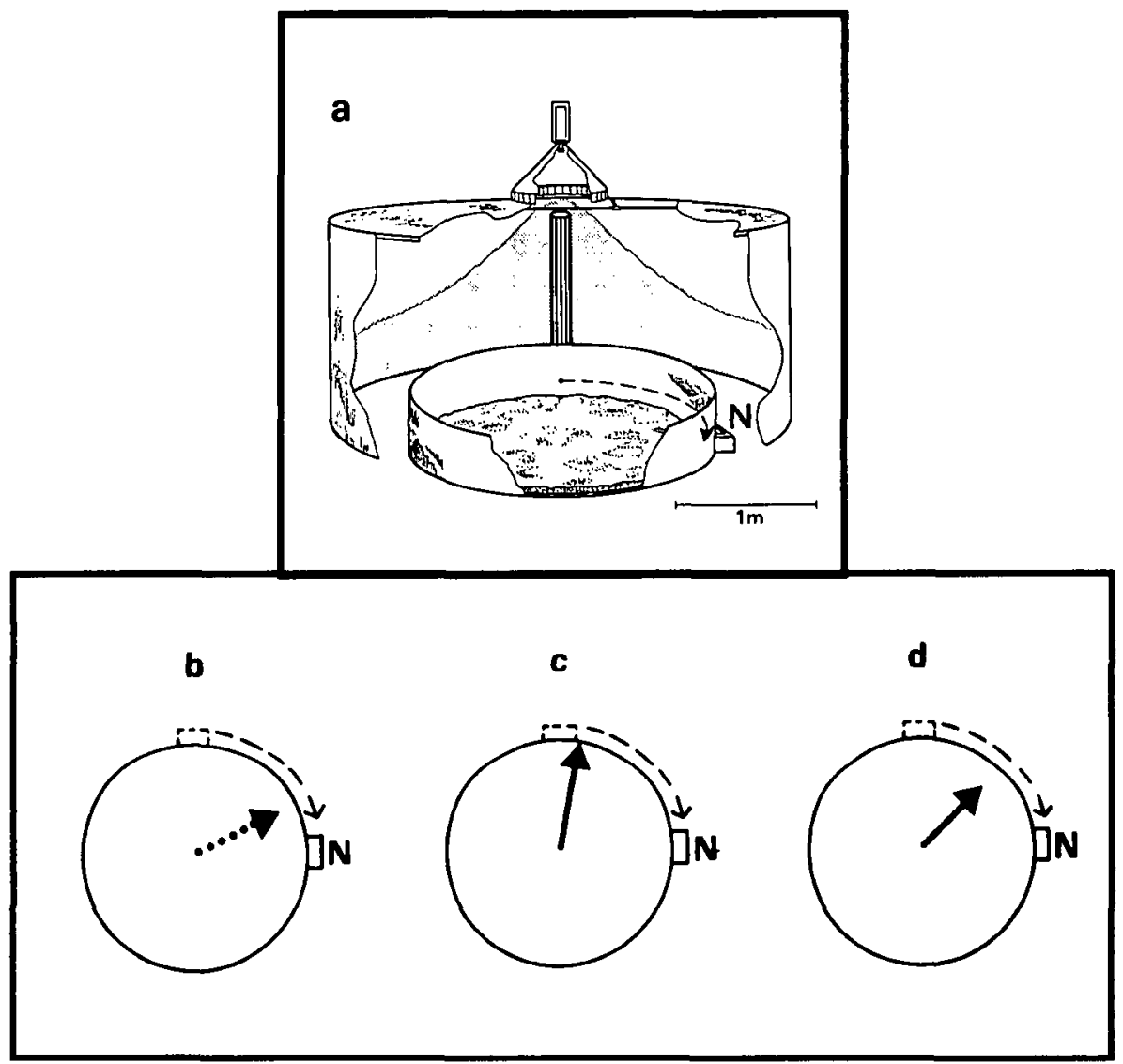

Fig. 3. The influence of landmarks presented within a symmetrical white enclosure. In its standard location the nest box is located at $0^{\circ}$, upwards. On all graphs, the arena and nest box, N, are rotated clockwise by $90^{\circ}$ before the subject leaves the nest exit. Graph (a) represents the circular arena within a symmetrical enclosure, and the 'optimal landscape', consisting of a black and white striped vertical cylinder $(r=8 \mathrm{~cm}, h=140 \mathrm{~cm})$ presented against a continuous black background pattern (wrapped Cauchy curve, $\rho=0.9$ ). Graph (b) represents the mean homing vector of 4 subjects $(P>0.05)$ when the landscape consists of a vertical cylinder only. Graphs (c) and (d) represent the mean homing vector of 6 subjects $(P<0.01$, Moore's test) when the landscape consists of the background pattern plus a vertical cylinder $(c)$ or of the same background pattern plus a horizontal cylinder $(d)$

So far, it seems that dead reckoning is mainly expressed in 'domicentred roundtrips'; i.e., in excusions where a sedentary animal leaves its home, the centre of its universe, and then returns again to it. The hypothesis that dead reckoning continuously informs the locomoting subject on its position and heading in the real and represented spatial environment is examined by a number of different test situations. According to our main procedures, the subject is trained to reach from its nest one or several food sources, which it may pinpoint by using a specific landmark. However, the relevant landmark represents one element within a set of identical landmarks, and therefore has to be identified by its position. Through dead reckoning, the hamster is informed on its own 
position, and this allows the animal to evaluate the position and therefore the identity of the relevant landmark. So far, our results are encouraging, ${ }^{9}$ but more evidence is needed.

5. ACKNOWLEDGMENTs. This work is supported by the Swiss NSF grant 31-26227.89. We wish to thank the members of the Laboratory of Ethology, University of Geneva, for their participation in the research.

\section{REFERENCES}

1 Bennett, A. T. D. (1993). A framework of spatial memory models. Paper presented at the Conference of the Royal Institute of Navigation, Oxford.

${ }^{2}$ Collett, T. S., Cartwright, B. A. and Smith, B. A. (1986). Landmark learning and visuospatial memories in gerbils. Journal of Comparative Physiology, 158, 835-851.

3 Etienne, A. S., Hurni, C., Maurer, R. and Séguinot, V. (1991). Twofold path integration during hoarding in the golden hamster? Ethology, Ecology and Evolution, 3, I-1 1.

4 Etienne, A. S., Joris, S., Maurer, R. and Teroni, E. (1990). Enhancing the impact of extramaze cues in a spatial orientation task. Behavioural Brain Research, 38, 199-2 10.

${ }^{5}$ Etienne, A. S., Maurer, R. and Saucy, F. (1988). Limitations in the assessment of pathdependent information. Behaviour, 106, 8 I- I I I.

${ }^{6}$ Etienne, A. S., Maurer, R., Saucy, F. and Teroni, E. (1986). Short-distance homing in the golden hamster after a passive outward journey. Animal Behaviour 34, 696-7 I 5 .

7 Etienne, A. S., Teroni, E., Hurni, C. and Portenier, V. (1990). The effect of a single light cue on homing behaviour of the golden hamster. Animal Behaviour, 39, 17-41.

8 Gallistel, C. R. The Organization of Learning (1990). MIT Press, Cambridge, MA.

${ }_{9}$ Georgakopoulos, J. and Etienne, A. S. Identifying location by dead reckoning and local cues. Behavioural Processes. (In the Press.)

${ }^{10}$ Maurer, R. (1993). Dead reckoning in animals: can PDP help us unravel the mystery of partial solutions? Paper presented at the Conference of the Royal Institute of Navigation, Oxford.

$"$ O'Keefe, J. and Nadel, L. (1978). The Hippocampus as a Cognitive Map. Clarendon Press, London.

12 Séguinot, V., Maurer, R. and Etienne, A.S. Dead reckoning in a small mammal: the evaluation of distance. Journal of Comparative Physiology. (In the Press.)

13 Teroni, E., Portenier, V. and Etienne, A. S. (1987). Spatial orientation of the golden hamster in conditions of conflicting location-based and route-based information. Behavioural Ecology and Sociobiology, 20, 389-397.

\section{KEY WORDS}
1. Animal navigation
2. Dead reckoning 